\title{
Prevention of pain during injection of microemulsion propofol: application of lidocaine mixture and the optimal dose of lidocaine
}

\author{
Hyun-Sik Kim, Kwang Rae Cho, Jeong Han Lee, Young Hwan Kim, Se Hun Lim, Kun Moo Lee, Soon
} Ho Cheong, Young Jae Kim, Chee-Mahn Shin, and Jin-Young Lee

Department of Anesthesiology and Pain Medicine, Busan Paik Hospital, College of Medicine, Inje University, Busan, Korea

Background: Similar to lipid emulsion propofol, microemulsion propofol also causes a high incidence of pain during intravenous injection. Various methods have been used to minimize the incidence and severity of pain on injection of lipid emulsion propofol. In this study, we investigated the effect of a lidocaine mixture on pain induced by microemulsion propofol injection, and sought to determine the optimal dose of lidocaine that could reduce pain on injecting a propofol-lidocaine mixture.

Methods: One hundred sixty $(n=160)$ patients of American Society of Anesthesiologists physical status class I or II were randomly allocated to four groups: Group A, control; Group B, 20 mg lidocaine; Group C, 30 mg lidocaine; Group D, 40 mg lidocaine. In each patient, pain on microemulsion propofol solution injection was graded as none, mild, moderate, or severe.

Results: The incidence of pain in groups A, B, C, and D was $97.5 \%, 80 \%, 65 \%$, and $50 \%$, respectively. Increasing the lidocaine dose significantly reduced pain $(\mathrm{P}<0.05)$. One patient in Group $\mathrm{D}(2.5 \%)$ had moderate to severe pain, which was significantly lower than groups $\mathrm{B}(42.5 \%)$ and $\mathrm{C}(32.5 \%)(\mathrm{P}<0.05)$.

Conclusions: The lidocaine and propofol mixture is effective in alleviating pain associated with microemulsion propofol injection. Within this dose range and in this patients population, increasing lidocaine dosage significantly reduced pain during injection of microemulsion propofol. (Korean J Anesthesiol 2010; 59: 310-313)

Key Words: Injection pain, Lidocaine, Microemulsion propofol.

Received: March 4, 2010. Revised: 1st, March 22, 2010; 2nd, April 17, 2010. Accepted: July 9, 2010.

Corresponding author: Kwang Rae Cho, M.D., Department of Anesthesiology and Pain Medicine, Busan Paik Hospital, College of Medicine, Inje University, Gaegeum 2-dong, Busanjin-gu, Busan 614-735, Korea. Tel: 82-51-890-6520, Fax: 82-51-898-4216, E-mail: maeteo@hanmir.com This work was supported by Grant from Inje University, 2009.

(c) This is an open-access article distributed under the terms of the Creative Commons Attribution Non-Commercial License (http:// creativecommons.org/licenses/by-nc/3.0/), which permits unrestricted non-commercial use, distribution, and reproduction in any medium, provided the original work is properly cited. 


\section{Introduction}

Lipid emulsion propofol (2,6-diisopropylphenol), an intravenous (IV) anesthetic, is commonly used for general anesthesia because of its rapid onset, short duration, and the excellent quality of recovery it provides. Nevertheless, lipid emulsion propofol has been associated with several drawbacks [1-3], and the incidence of pain secondary to lipid emulsion propofol injection varies from $59.1 \%$ to $100 \%$, when injection is made into a vein on the dorsum of the hand $[4,5]$.

Lipid-free microemulsion propofol (Aquafol ${ }^{\mathrm{TM}}$, Daewon Pharmaceutical Co., Ltd., Seoul, Korea) was newly developed to reduce the risk of lipid solvent-related adverse drug reactions. However, pain on the injection of microemulsion propofol is still an unresolved problem [6], which not only causes distresses in patients, but also present challenges to the anesthesiologists.

The mechanism of pain on propofol injection remains unclear and requires further investigations. Although various methods have been attempted to attenuate this injection pain [4,7-17], adding lidocaine to propofol is the most popular method.

In the present study, we sought to evaluate the efficacy of lidocaine in minimizing microemulsion propofol injection pain, and to determine the optimal dose of lidocaine to be added to microemulsion propofol.

\section{Materials and Methods}

The Institutional Review Board of our institution approved the study protocol, and written consent was obtained from all patients prior to study enrollment. One hundred sixty ( $\mathrm{n}=$ 160) American Society of Anesthesiologists physical status I and II patients, aged 16-65 years, and scheduled for elective surgery, were selected. Patients with allergies to any drugs or renal, hepatic, or cardiac problems, neurologic deficits or psychiatric disorders were excluded. None of the patients was premedicated before entering the operation room, and all had a 20-gauge cannula placed into a vein on the dorsum of the nondominant hand. Standard monitoring, including electrocardiography, noninvasive arterial pressure, pulse oximetry, capnography, and body temperature, was performed throughout the procedure.

Patients were randomly allocated into one of four groups. Patients in Group A were injected intravenously with $12 \mathrm{ml}$ of microemulsion propofol plus $3 \mathrm{ml}$ of saline. Patients in Group B were injected intravenously with $12 \mathrm{ml}$ of microemulsion propofol, $2 \mathrm{ml}$ of saline, and $1 \mathrm{ml}$ of $2 \%$ lidocaine $(20 \mathrm{mg}$ ). Patients in Group C were injected intravenously with $12 \mathrm{ml}$ of microemulsion propofol, $1.5 \mathrm{ml}$ of saline, and $1.5 \mathrm{ml}$ of $2 \%$ lidocaine (30 mg). Patients in Group D were injected intravenously with $12 \mathrm{ml}$ of microemulsion propofol, $1 \mathrm{ml}$ of saline, and $2 \mathrm{ml}$ of $2 \%$ lidocaine ( $40 \mathrm{mg}$ ). No significant difference was observed in patient characteristics (Table 1).

Propofol was drawn up into a polyethylene syringe by an assistant and made up to a total volume of $15 \mathrm{ml}$ using saline, as necessary. All drugs were kept at room temperature and administered within 20 minutes of preparation. In a doubleblind manner, propofol solution was manually injected by an anesthesiologist at a rate of $3 \mathrm{ml}$ every 5 seconds until the patient lost consciousness. All patients were questioned every 5 seconds during the injection regarding pain or discomfort in the arm. The scale of pain was then recorded. The pain scale was defined as follows: no pain; mild pain (tolerable soreness or slight pain); moderate pain (subjective complaint between mild and severe pain); severe pain (pain causing the patient to flex his/her arm to deny injection). If the patient lost consciousness before injecting $15 \mathrm{ml}$ of the solution, the remaining portion would not be administered. We also recorded the time losing consciousness. After recovering from anesthesia, patients were asked if they had any recollection of discomfort or pain during the induction period.

Statistical analyses were performed using SPSS (version 17.0, SPSS Inc., Chicago, IL, USA). The differences in mean painintensity scores among the groups were analyzed with the chisquare test for trends. The time of unconsciousness among the groups did not follow a normal distribution, and were analyzed with one-way ANOVA using log-transformed data, followed by Scheffe post hoc test. $\mathrm{P}$ value $<0.05$ was considered statistically significant.

Table 1. Demographic Data

\begin{tabular}{lccc}
\hline & $\mathrm{A}(\mathrm{n}=40)$ & $\mathrm{B}(\mathrm{n}=40)$ & $\mathrm{C}(\mathrm{n}=40)$ \\
\hline Age (yr) & $40.9 \pm 13.0$ & $37.9 \pm 14.7$ & $42.2 \pm 13.4$ \\
Weight (kg) & $65.6 \pm 12.0$ & $63.5 \pm 8.2$ & $63.2 \pm 11.3$ \\
Height (cm) & $168.9 \pm 9.3$ & $166.4 \pm 7.6$ & $165.5 \pm 8.7$ \\
Gender (M/F) & $25 / 15$ & $23 / 17$ & $20 / 20$ \\
ASA (I/II) & $33 / 7$ & $34 / 6$ & $28 / 12$ \\
\hline
\end{tabular}

Values are presented as means \pm SD. Group A receive $12 \mathrm{ml}$ of microemulsion propofol plus $3 \mathrm{ml}$ of saline, Group B receive $12 \mathrm{ml}$ of microemulsion propofol, $2 \mathrm{ml}$ of saline, and $1 \mathrm{ml}$ of $2 \%$ lidocaine $(20 \mathrm{mg})$, Group C receive $12 \mathrm{ml}$ of microemulsion propofol, and $1.5 \mathrm{ml}$ of saline, and $1.5 \mathrm{ml}$ of $2 \%$ lidocaine ( $30 \mathrm{mg}$ ), Group D receive $12 \mathrm{ml}$ of microemulsion propofol, $1 \mathrm{ml}$ of saline, and $2 \mathrm{ml}$ of $2 \%$ lidocaine (40 mg). There is no significant difference between the groups. ASA: American Society of Anesthesiologists Physical Status. 
Table 2. Incidence of Pain Reported at the Time of Anesthesia Induction

\begin{tabular}{ccccc}
\hline Group & $\begin{array}{c}\text { No pain } \\
\mathrm{n}(\%)\end{array}$ & $\begin{array}{c}\text { Mild } \\
\mathrm{n}(\%)\end{array}$ & $\begin{array}{c}\text { Moderate } \\
\mathrm{n}(\%)\end{array}$ & $\begin{array}{c}\text { Severe } \\
\mathrm{n}(\%)\end{array}$ \\
\hline $\mathrm{A}$ & $1(2.5)$ & $5(12.5)$ & $12(30.0)$ & $22(55.0)$ \\
$\mathrm{B}$ & $8(20.0)$ & $15(37.5)$ & $12(30.0)$ & $5(12.5)$ \\
$\mathrm{C}$ & $14(35.0)$ & $13(32.5)$ & $10(25.0)$ & $3(7.5)$ \\
$\mathrm{D}$ & $20(50.0)$ & $19(47.5)$ & $0(0.0)$ & $1(2.5)$ \\
\hline
\end{tabular}

Group Areceive $12 \mathrm{ml}$ of microemulsion propofol plus $3 \mathrm{ml}$ of saline, Group B receive $12 \mathrm{ml}$ of microemulsion propofol, $2 \mathrm{ml}$ of saline, and $1 \mathrm{ml}$ of $2 \%$ lidocaine ( $20 \mathrm{mg}$ ), Group C receive $12 \mathrm{ml}$ of microemulsion propofol, and $1.5 \mathrm{ml}$ of saline, and $1.5 \mathrm{ml}$ of $2 \%$ lidocaine $(30 \mathrm{mg}$ ), Group D receive $12 \mathrm{ml}$ of microemulsion propofol, $1 \mathrm{ml}$ of saline, and $2 \mathrm{ml}$ of $2 \%$ lidocaine $(40 \mathrm{mg}$ ).

\section{Results}

The number of patients in each group who experienced pain or discomfort is shown in Table 2. The incidence of pain in groups A (control), B, C, and D was $97.5 \%, 80 \%, 65 \%$, and $50 \%$, respectively. Increasing the lidocaine dosage significantly reduced pain $(\mathrm{P}<0.05)$. The incidence of moderate to severe pain was $85 \%$ in the control group and that in the other groups was $42.5 \%$ (Group B), 32.5\% (Group C), and 2.5\% (Group D) (P $<0.05)$.

Loss of consciousness was more rapid in Group A than in all other groups $(\mathrm{P}<0.05$, Table 3$)$. In the recovery room, only one patient who reported pain was unable to recall pain scale and, all patients except one reported the same pain scale at the time of anesthesia induction.

\section{Discussion}

Microemulsion propofol, consists of $1 \%$ propofol, $8 \%$ polyethylene glycol 660 hydroxystearate (Solutol HS 15, BASF Co., Ltd., Seoul, Korea), and 5\% tetrahydrofurfuryl alcohol polyethylene glycol ether (Glycofurol, Roche, Basle, Switzerland), is thermodynamically stable, because it does not contain a long chain triglyceride emulsion, unlike the original lipid emulsion propofol. This formulation avoids the risk of lipid solvent-related adverse drug reactions, such as fat embolism, postoperative infection, hypertriglyceridemia and pancreatitis [1-3]. However, similar to other existing propofol formulations, newly developed microemulsion propofol still causes pain on injection. In addition, microemulsion propofol produces more frequent (69.7-89\%) and severe pain (51\%) upon injection than long-chain triglyceride propofol $[6,18]$. In our study the overall incidence of pain was $97.5 \%$, and $55 \%$ of patient experienced severe pain.

The concentration of free propofol in the aqueous phase and activation of the enzymatic cascade of the plasma kallikrein-
Table 3. The Time of Unconsciousness

\begin{tabular}{cc}
\hline Group & Time of unconsciousness (sec) \\
\hline A & $40.2 \pm 16.0$ \\
B & $46.0 \pm 10.1^{*}$ \\
C & $52.6 \pm 12.7^{*}$ \\
D & $48.3 \pm 9.0^{*}$ \\
\hline
\end{tabular}

Values are presented as mean \pm SD. Group Areceive $12 \mathrm{ml}$ of microemulsion propofol plus $3 \mathrm{ml}$ of saline, Group B receive $12 \mathrm{ml}$ of microemulsion propofol, $2 \mathrm{ml}$ of saline, and $1 \mathrm{ml}$ of $2 \%$ lidocaine (20 $\mathrm{mg}$ ), Group C receive $12 \mathrm{ml}$ of microemulsion propofol, and $1.5 \mathrm{ml}$ of saline, and $1.5 \mathrm{ml}$ of $2 \%$ lidocaine ( $30 \mathrm{mg}$ ), Group D receive $12 \mathrm{ml}$ of microemulsion propofol, $1 \mathrm{ml}$ of saline, and $2 \mathrm{ml}$ of $2 \%$ lidocaine (40 mg). $* \mathrm{P}<0.05$ compared with Group A.

kinin system are associated with the intensity of pain upon injection $[19,20]$. Higher aqueous free propofol concentrations of microemulsion propofol produce more frequent and severe pain [6]. However, the mechanism of pain upon injection of propofol is still unclear and requires further investigation. Various methods have been attempted to attenuate this injection pain. They include prior administration of opioid [7], lidocaine [7], prilocaine [8], thiopental [9], metocloproamide [10], ondansetron [11], ephedrine [12], the addition of 10-40 mg lidocaine to the propofol emulsion $[4,13,14]$, cooling the propofol to $4^{\circ} \mathrm{C}$ before injection [15], and making use of the antecubital fossa vein for injection [16]. These methods do not eradicate pain completely, but do decrease the incidence or severity of injection pain. To date, adding lidocaine to propofol is the most popular method to reduce injection pain of lipid emulsion propofol. Indeed, $30 \mathrm{mg}$ of lidocaine is the optimal dose typically used to reduce pain on injection [17]. Lidocaine mixed with lipid emulsion propofol reduces its $\mathrm{pH}$, lowers propofol concentration in the aqueous phase, and results in reduced pain [21]. Lidocaine mixed with propofol is more effective than pretreatment with lidocaine for decreasing propofol injection pain [16]. The $\mathrm{pH}$ of microemulsion propofol $(7.51 \pm 0.01)$ is similar to that of the lipid emulsion, and aqueous free propofol concentration is seven times higher than that of lipid emulsion [6].

It is well accepted that injecting through a large vein, such as the antecubital fossa vein, is an effective way to reduce pain on propofol injection. This is presumably because the drug contacts the mid-stream in the venous lumen and due to reduced contact with the sensitive vein wall. The drug may also be buffered effectively by the blood, with which it can mix freely [16]. Nonetheless, a cannula is usually placed into a vein on the dorsum of the nondominant hand, because many anesthesiologists prefer ease of access, ease of detecting extravasation, and there is a low incidence of neural or arterial damage. Premedications, such as benzodiazepine, analgesic 
opioid, and anticholinergics appears to have little effect on the incidence of pain during propofol injection [22-24], but other premedications, such as hydroxyzine may reduce pain severity [25], so we did not premedicate.

Lidocaine may induce cardiovascular, neurotoxic and other complications. However, less than $1 \mathrm{mg} / \mathrm{kg}$ can be used safely [26]. Thus, our study applied 20-40 mg of lidocaine. The results of this study show the effect of adding lidocaine to microemulsion propofol in reducing pain during injection.

More study is needed, as the loss of consciousness was more rapid in Group A than in all other groups. Similar to our findings, two other studies suggested decreased anesthetic potency of propofol when administered as lidocaine mixed with propofol in rats [27] and women [10]. In contrast to our finding, two studies showed clinically insignificant effects in the study ranged from $200: 10$ to $200: 50$ propofol/lidocaine mg ratio [28] and $10: 1$ propofol $1 \% /$ lodocaine $1 \%$ volume ratio [29].

In conclusion, a lidocaine and propofol mixture is effective in alleviating pain associated with microemulsion propofol injection. Within this dose range and in this patients population, increasing lidocaine dosage significantly reduced pain during injection of microemulsion propofol.

\section{References}

1. Bennett SN, McNeil MM, Bland LA, Arduino MJ, Villarino ME, Perrotta DM, et al. Postoperative infections traced to contamination of an intravenous anesthetic, propofol. N Engl J Med 1995; 333: 14754.

2. Devlin JW, Lau AK, Tanios MA. Propofol-associated hypertriglyceridemia and pancreatitis in the intensive care unit: an analysis of frequency and risk factors. Pharmacotherapy 2005; 25: 1348-52.

3. Kim KM, Choi BM, Park SW, Lee SH, Christensen LV, Zhou J, et al. Pharmacokinetics and pharmacodynamics of propofol microemulsion and lipid emulsion after an intravenous bolus and variable rate infusion. Anesthesiology 2007; 106: 924-34.

4. Johnson RA, Harper NJ, Chadwick S, Vohra A. Pain on injection of propofol: methods of alleviation. Anaesthesia 1990; 45: 439-42.

5. Hynynen M, Korttila K, Tammisto T. Pain on i.v. injection of propofol (ICI 35 868) in emulsion formulation. Short communication. Acta Anaesthesiol Scand 1985; 29: 651-2.

6. Sim JY, Lee SH, Park DY, Jung JA, Ki KH, Lee DH, et al. Pain on injection with microemulsion propofol. Br J Clin Pharmacol 2009; 67:316-25.

7. Kobayashi Y, Naganuma R, Seki S, Aketa K, Ichimiya T, Namiki A. Reduction of pain on injection of propofol: a comparison of fentanyl with lidocaine. Masui 1998; 47: 963-7.

8. Eriksson M. Prilocaine reduces injection pain caused by propofol. Acta Anaesthesiol Scand 1995; 39: 210-3.

9. Agarwal A, Ansari MF, Gupta D, Pandey R, Raza M, Singh PK, et al.
Pretreatment with thiopental for prevention of pain associated with propofol injection. Anesth Analg 2004; 98: 683-6.

10. Fujii Y, Uemura A. Effect of metoclopramide on pain on injection of propofol. Anaesth Intensive Care 2004; 32: 653-6.

11. Ambesh SP, Dubey PK, Sinha PK. Ondansetron pretreatment to alleviate pain on propofol injection: a randomized, controlled, double-blinded study. Anesth Analg 1999; 89: 197-9.

12. Cheong MA, Kim KS, Choi WJ. Ephedrine reduces the pain from propofol injection. Anesth Analg 2002; 95: 1293-6.

13. Gehan G, Karoubi P, Quinet F, Leroy A, Rathat C, Pourriat JL. Optimal dose of lignocaine for preventing pain on injection of propofol. Br J Anaesth 1991; 66: 324-6.

14. Helbo-Hansen S, Westergaard V, Krogh BL, Svendsen HP. The reduction of pain on injection of propofol: the effect of addition of lignocaine. Acta Anaesthesiol Scand 1988; 32: 502-4.

15. McCrirrick A, Hunter S. Pain on injection of propofol: the effect of injectate temperature. Anaesthesia 1990; 45: 443-4.

16. Scott RP, Saunders DA, Norman J. Propofol: clinical strategies for preventing the pain of injection. Anaesthesia 1988; 43: 492-4.

17. Gajraj NM, Nathanson MH. Preventing pain during injection of propofol: the optimal dose of lidocaine. J Clin Anesth 1996; 8: 575-7.

18. Dubey PK, Kumar A. Pain on injection of lipid-free propofol and propofol emulsion containing medium-chain triglyceride: a comparative study. Anesth Analg 2005; 101: 1060-2.

19. Klement W, Arndt JO. Pain on injection of propofol: effects of concentration and diluent. Br J Anaesth 1991; 67: 281-4.

20. Doenicke AW, Roizen MF, Rau J, Kellermann W, Babl J. Reducing pain during propofol injection: the role of the solvent. Anesth Analg 1996; 82: 472-4.

21. Eriksson M, Englesson S, Niklasson F, Hartvig P. Effect of lignocaine and $\mathrm{pH}$ on propofol-induced pain. Br J Anaesth 1997; 78: 502-6.

22. Fragen RJ, de Grood PM, Robertson EN, Booij LH, Crul JF. Effects of premedication on diprivan induction. Br J Anaesth 1982; 54: 913-6.

23. Briggs LP, Bahar M, Beers HT, Clarke RS, Dundee JW, Wright PJ, et al. Effect of preanaesthetic medication on anaesthesia with ICI 35, 868. Br J Anaesth 1982; 54: 303-6.

24. Briggs LP, White M. The effects of premedication on anaesthesia with propofol ('Diprivan'). Postgrad Med J 1985; 61 Suppl 3: 35-7.

25. Bilaine J, Desmonts JM. Effect of premedication with atropine or hydroxyzine on induction and maintenance of anaesthesia with propofol ('Diprivan'). Postgrad Med J 1985; 61 Suppl 3: 38-9.

26. Nightingale P, Healy TE, Hargreaves J, McGuinness K, Kay B. Propofol in emulsion form: induction characteristics and venous sequelae. Eur J Anaesthesiol 1985; 2: 361-8.

27. Eriksson M, Englesson S, Hörte I, Hartvig P. The anaesthetic potency of propofol in the rat is reduced by simultaneous intravenous administration of lignocaine. Eur J Anaesthesiol 1999; 16: 315-9.

28. Lilley EM, Isert PR, Carasso ML, Kennedy RA. The effect of the addition of lignocaine on propofol emulsion stability. Anaesthesia 1996; 51: 815-8.

29. Tan LH, Hwang NC. The effect of mixing lidocaine with propofol on the dose of propofol required for induction of anesthesia. Anesth Analg 2003; 97: 461-4. 